\title{
Positive Radial Solutions for a Class of Semilinear Elliptic Problems Involving Critical Hardy-Sobolev Exponent and Hardy Terms
}

\author{
Yong-Yi Lan \\ School of Sciences, Jimei University, Xiamen, China \\ Email: 200661000135@jmu.edu.cn
}

How to cite this paper: Lan, Y.-Y. (2017) Positive Radial Solutions for a Class of Semilinear Elliptic Problems Involving Critical Hardy-Sobolev Exponent and Hardy Terms. Journal of Applied Mathematics and Physics, 5, 2205-2217. https://doi.org/10.4236/jamp.2017.511180

Received: September 6, 2017

Accepted: November 14, 2017

Published: November 17, 2017

Copyright $\odot 2017$ by author and Scientific Research Publishing Inc. This work is licensed under the Creative Commons Attribution International License (CC BY 4.0).

http://creativecommons.org/licenses/by/4.0/

\section{Open Access}

\begin{abstract}
In this paper, we investigate the solvability of a class of semilinear elliptic equations which are perturbation of the problems involving critical Hardy-Sobolev exponent and Hardy singular terms. The existence of at least a positive radial solution is established for a class of semilinear elliptic problems involving critical Hardy-Sobolev exponent and Hardy terms. The main tools are variational method, critical point theory and some analysis techniques.
\end{abstract}

\section{Keywords}

Hardy Singular Terms, Hardy-Sobolev Exponent, Positive Radial Solution, Perturbation Method, Variational Approach

\section{Introduction and Main Results}

In this paper, we are concerned with the existence of positive radial solutions for the following semilinear elliptic problem with Hardy-Sobolev exponent and Hardy singular terms:

$$
\begin{cases}-\Delta u-\mu \frac{u}{|x|^{2}}=[1+\delta h(|x|)] \frac{|u|^{2^{*}(s)-2}}{|x|^{s}} u, & x \in \mathbb{R}^{N} \\ u>0, & x \in \mathbb{R}^{N} \\ u \in D_{r}^{1,2}\left(\mathbb{R}^{N}\right)=\left\{u \in D^{1,2}\left(\mathbb{R}^{N}\right): u \text { is radial }\right\}, & \end{cases}
$$

where $0<s<2,2^{*}(s)=\frac{2(N-s)}{N-2}$ is the Hardy-Sobolev critical exponent and $2^{*}=2^{*}(0)=\frac{2 N}{N-2}$ is the Sobolev critical exponent, $\mu<\bar{\mu} \triangleq \frac{(N-2)^{2}}{4}$. 
$D^{1,2}\left(\mathbb{R}^{N}\right)(N \geq 3)$ denotes the space of the functions $u \in L^{2^{*}}\left(\mathbb{R}^{N}\right)$ such that $\nabla u \in L^{2}\left(\mathbb{R}^{N}\right)$, endowed with scalar product and norm, respectively, given by

$$
\begin{aligned}
& \langle u, v\rangle=\int_{\mathbb{R}^{N}}\left(\nabla u \cdot \nabla v-\mu \frac{u v}{|x|^{2}}\right) \mathrm{d} x, \\
& \|u\|^{2}=\int_{\mathbb{R}^{N}}\left(|\nabla u|^{2}-\mu \frac{u^{2}}{|x|^{2}}\right) \mathrm{d} x,
\end{aligned}
$$

that coincides with the completion of $C_{0}^{\infty}\left(\mathbb{R}^{N}\right)$ with respect to the $L^{2}$-norm of the gradient. By Hardy inequality [1], we easily derive that the norm is equivalent to the usual norm:

$$
\|u\|_{0}^{2}=\int_{\mathbb{R}^{N}}|\nabla u|^{2} \mathrm{~d} x
$$

in $D^{1,2}\left(\mathbb{R}^{N}\right)$.

Clearly, $D_{r}^{1,2}\left(\mathbb{R}^{N}\right)$ is a closed subset of $D^{1,2}\left(\mathbb{R}^{N}\right)$, so $D_{r}^{1,2}\left(\mathbb{R}^{N}\right)$ is a Hilbert space. By the symmetric criticality principle, in view of [2], we know that the positive radial solutions of problem (1.1) correspond to the nonzero critical points of the functional $I_{\delta}: D_{\mathrm{r}}^{1,2}\left(\mathbb{R}^{N}\right) \rightarrow \mathbb{R}$ defined by

$$
\begin{aligned}
I_{\delta}(u)= & \frac{1}{2} \int_{\mathbb{R}^{N}}\left(|\nabla u|^{2}-\mu \frac{u^{2}}{|x|^{2}}\right) \mathrm{d} x-\frac{1}{2^{*}(s)} \int_{\mathbb{R}^{N}} \frac{\left|u^{+}\right|^{2^{*}(s)}}{|x|^{s}} \mathrm{~d} x \\
& -\frac{\delta}{2^{*}(s)} \int_{\mathbb{R}^{N}} h(|x|) \frac{\left|u^{+}\right|^{2^{*}(s)}}{|x|^{s}} \mathrm{~d} x,
\end{aligned}
$$

where $u^{+}=\max \{u, 0\}$.

The reason why we investigate (1.1) is the presence of the Hardy-Sobolev exponent, the unbounded domain $\mathbb{R}^{N}$ and the so-called inverse square potential in the linear part, which cause the loss of compactness of embedding $D^{1,2}\left(\mathbb{R}^{N}\right) \rightarrow L^{2^{*}}\left(\mathbb{R}^{N}\right), \quad H^{1}\left(\mathbb{R}^{N}\right) \rightarrow L^{p}\left(\mathbb{R}^{N}\right) \quad$ and $\quad D^{1,2}\left(\mathbb{R}^{N}\right) \rightarrow L^{2}\left(|x|^{-2} \mathrm{~d} x\right)$. Hence, we face a type of triple loss of compactness whose interacting with each other will result in some new difficulties. In last two decades, loss of compactness leads to many interesting existence and nonexistence phenomena for elliptic equations. There are abundant results about this class of problems. For example, by using the concentration compactness principle, the strong maximum principle and the Mountain Pass lemma, Li et al. [3] had obtained the existence of positive solutions for singular elliptic equations with mixed Dirichlet-Neumann boundary conditions involving Sobolev-Hardy critical exponents and Hardy terms. Bouchekif and Messirdi [4] obtained the existence of positive solution to the elliptic problem involving two different critical Hardy-Sobolev exponents at the same pole by variational methods and concentration compactness principle. Lan and Tang [5] have obtained some existence results of (1.1) with $\mu=0$ via an abstract perturbation method in critical point theory. There are some other sufficient conditions, we refer the 
interested readers to ([6]-[18]) and the references therein.

In the present paper, we investigate the existence of positive radial solutions of problem (1.1). There are several difficulties in facing this problem by means of variational methods. In addition to the lack of compactness, there are more intrinsic obstructions involving the nature of its critical points. Based on a suitable use of an abstract perturbation method in critical point theory discussed in [5] [13] [14], we show that the semilinear elliptic problem with Hardy-Sobolev exponent and Hardy singular terms has at least a positive radial solution.

In this paper, we assume that $h$ satisfies one of the following conditions:

(H) $h \in L^{\infty}\left(\mathbb{R}^{N}\right) \cap C^{1}\left(\mathbb{R}^{N}\right), h(x)=h(|x|)=h(r), r=|x|$, and

$$
\int_{1}^{\infty} r^{-\alpha+N-s-1} h(r) \mathrm{d} r<\infty
$$

for some $\alpha<N-S$.

$\left(\mathrm{H}^{\prime}\right) \quad h \in C^{2}\left(\mathbb{R}^{N}\right), h(x)=h(|x|)=h(r), r=|x|, h(r)$ is $T$-periodic and

$$
\int_{0}^{T} h(r) \mathrm{d} r=0
$$

The main results read as follows.

Theorem 1 Let $(\mathrm{H})$ hold, and assume that $h(0)=0$ and $h \not \equiv 0$. Then for $|\delta|$ small, problem (1.1) has a positive radial solution $u_{\delta}$.

Remark 1 It is easy to check that the following function $h(r)$ satisfies the conditions of Theorem 1 ,

$$
h(r)=\frac{2 r}{\mathrm{e}^{r}} .
$$

Theorem 2 If assumption (H) holds, and suppose that $h \in C^{2}\left(\mathbb{R}^{N}\right)$ and $h(0) h^{\prime \prime}(0)>0$. Then for $|\delta|$ small, problem (1.1) has a positive radial solution $u_{\delta}$.

Remark 2 It is easy to check that the following function $h(r)$ satisfies the conditions of Theorem 2,

$$
h(r)=\frac{1-2 r}{\mathrm{e}^{r}} .
$$

Theorem 3 Assume that $(\mathrm{H})$ holds, and suppose

$$
\int_{0}^{\infty} h(r)\left(1+r^{2-s}\right)^{-\frac{2(N-s)}{2-s}} r^{N-s-1} \mathrm{~d} r \neq 0
$$

and $\int_{0}^{\infty} h(0) h(r)\left(1+r^{2-s}\right)^{-\frac{2(N-s)}{2-s}} r^{N-s-1} \mathrm{~d} r \leq 0$.

Then for $|\delta|$ small, problem (1.1) has a positive radial solution $u_{\delta}$.

Remark 3 It is easy to check that the following function $h(r)$ satisfies the conditions of Theorem 3 for all $N \geq 3$ and $0<s<2$,

$$
h(r)=\frac{r}{\mathrm{e}^{r}}
$$

in fact, 


$$
\int_{0}^{\infty} h(r)\left(1+r^{2-s}\right)^{-\frac{2(N-s)}{2-s}} r^{N-s-1} \mathrm{~d} r \neq 0
$$

and $\int_{0}^{\infty} h(0) h(r)\left(1+r^{2-s}\right)^{-\frac{2(N-s)}{2-s}} r^{N-s-1} \mathrm{~d} r=0$;

We can also give the following example for $N=3$ and $s=1$,

$$
h(r)=\frac{1-100 r}{\mathrm{e}^{r}}
$$

in fact, with the help of computers, we can get

$$
\begin{aligned}
& \qquad \int_{0}^{\infty} \frac{1-100 r}{\mathrm{e}^{r}}\left(1+r^{2-1}\right)^{-\frac{2(3-1)}{2-1}} r^{3-1-1} \mathrm{~d} r \approx-4.06 \neq 0 \\
& \text { and } \int_{0}^{\infty} h(0) \frac{1-100 r}{\mathrm{e}^{r}}\left(1+r^{2-1}\right)^{-\frac{2(3-1)}{2-1}} r^{3-1-1} \mathrm{~d} r \approx-4.06<0 .
\end{aligned}
$$

Theorem 4 Suppose that assumption ( $\left.\mathrm{H}^{\prime}\right)$ holds, and satisfies the condition that $h(0) h^{\prime \prime}(0)>0$. Then problem (1.1) has a positive radial solution $u_{\delta}$, provided $|\delta| \ll 1$.

Remark 4 It is easy to check that the following function $h(r)$ satisfies the conditions of Theorem 4,

$$
h(r)=\mathrm{e}^{\sin \left(\frac{7 \pi}{4}+r\right)} \cos \left(\frac{7 \pi}{4}+r\right)
$$

in fact,

$$
h(0)=\mathrm{e}^{\sin \left(\frac{7 \pi}{4}+0\right)} \cos \left(\frac{7 \pi}{4}+0\right)=\frac{\sqrt{2}}{2} \mathrm{e}^{-\frac{\sqrt{2}}{2}}>0,
$$

and by a direct computation, we have

$$
h^{\prime \prime}(0)=\sqrt{2} \mathrm{e}^{-\frac{\sqrt{2}}{2}}>0 .
$$

Theorem 5 Let $h$ satisfy (H'), and suppose that $h(0)=0$ and $h \neq \equiv$. Then problem (1.1) has a positive radial solution $u_{\delta}$, provided $|\delta| \ll 1$.

Remark 5 It is easy to check that the following function $h(r)$ satisfies the conditions of Theorem 5 ,

$$
h(r)=\sin 2 r .
$$

This paper is organized as follows. After a first section we devoted to studying the unperturbed problem $-\Delta u-\mu \frac{u}{|x|^{2}}=\frac{|u|^{2^{*}(s)-2}}{|x|^{5}} u$. The main results are proved in Section 3. In the following discussion, we denote various positive constants as $C$ or $C_{i}(i=0,1,2,3, \cdots)$ for convenience. $o(t)$ denote $\frac{o(t)}{t} \rightarrow 0$ as $t \rightarrow 0^{+}$. This idea is essentially introduced in [5] [13].

\section{The Case $\delta=0$}

In this section, we will study the unperturbed problem 


$$
\left\{\begin{array}{l}
-\Delta u-\mu \frac{u}{|x|^{2}}=\frac{|u|^{2^{*}(s)-2}}{|x|^{s}} u, \quad x \in \mathbb{R}^{N} \\
u \in D_{r}^{1,2}\left(\mathbb{R}^{N}\right), \quad u>0, \quad x \in \mathbb{R}^{N} .
\end{array}\right.
$$

It is well-known that the nontrivial solutions of problem (2.1) are equivalent to the nonzero critical points of the energy functional

$$
I_{0}(u)=\frac{1}{2} \int_{\mathbb{R}^{N}}\left(|\nabla u|^{2}-\mu \frac{u^{2}}{|x|^{2}}\right) \mathrm{d} x-\frac{1}{2^{*}(s)} \int_{\mathbb{R}^{N}} \frac{\left|u^{+}\right|^{2^{*}(s)}}{|x|^{s}} \mathrm{~d} x, \quad u \in D_{r}^{1,2}\left(\mathbb{R}^{N}\right) .
$$

Obviously, the energy functional $I_{0}(u)$ is well-defined and is of $C^{2}$ with derivatives given by

$$
\begin{gathered}
\left\langle I_{0}^{\prime}(u), v\right\rangle=\int_{\mathbb{R}^{N}}\left(\nabla u \cdot \nabla v-\mu \frac{u v}{|x|^{2}}\right) \mathrm{d} x-\int_{\mathbb{R}^{N}} \frac{\left|u^{+}\right|^{2^{*}(s)-1}}{|x|^{s}} v \mathrm{~d} x, u, v \in D_{r}^{1,2}\left(\mathbb{R}^{N}\right) ; \\
\left\langle I_{0}^{\prime \prime}(u) v, w\right\rangle=\int_{\mathbb{R}^{N}}\left(\nabla v \cdot \nabla w-\mu \frac{v w}{|x|^{2}}\right) \mathrm{d} x-\int_{\mathbb{R}^{N}} \frac{\left(2^{*}(s)-1\right)\left|u^{+}\right|^{2^{*}(s)-2}}{|x|^{s}} v w \mathrm{~d} x \\
u, v, w \in D_{r}^{1,2}\left(\mathbb{R}^{N}\right) .
\end{gathered}
$$

For all $\varepsilon>0$, it is well known that the function

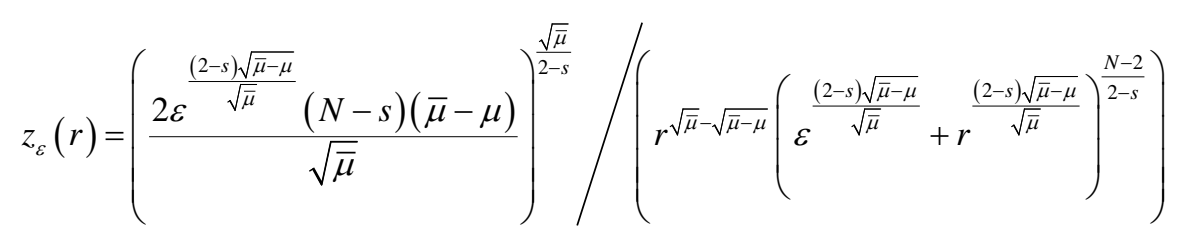

solves the equation (2.1) and satisfies

$$
\int_{\mathbb{R}^{N}}\left(\left|\nabla z_{\varepsilon}\right|^{2}-\mu \frac{z_{\varepsilon}^{2}}{|x|^{2}}\right) \mathrm{d} x=\int_{\mathbb{R}^{N}} \frac{\left|z_{\varepsilon}\right|^{2^{*}(s)}}{|x|^{s}} \mathrm{~d} x
$$

Let

$$
U(r)=\left(\frac{2(N-s)(\bar{\mu}-\mu)}{\sqrt{\bar{\mu}}}\right)^{\frac{\sqrt{\mu}}{2-s}} /\left(r^{\sqrt{\mu}-\sqrt{\bar{\mu}-\mu}}\left(1+r^{\frac{(2-s) \sqrt{\bar{\mu}-\mu}}{\sqrt{\bar{\mu}}}}\right)^{\frac{N-2}{2-s}}\right)
$$

then

$$
Z_{\varepsilon}(r)=\varepsilon^{-\frac{N-2}{2}} U\left(\frac{r}{\varepsilon}\right)
$$

$I_{0}$ has a (non-compact) 1-dimensional critical manifold given by

$$
Z=\left\{z=z_{\varepsilon}(r): \varepsilon>0\right\} .
$$

The unperturbed problem is invariant under the transformation that transforms the function $u(r)$ in the function $\varepsilon^{-\frac{N-2}{2}} u\left(\frac{r}{\varepsilon}\right)$. The purpose of this 
section is to show the following lemmas.

Lemma 2.1. For all $\varepsilon>0, T_{z_{\varepsilon}} Z=\operatorname{Ker}\left[I_{0}^{\prime \prime}\left(z_{\varepsilon}\right)\right]$.

Proof. We will prove the lemma by taking $\varepsilon=1$, hence $Z_{\varepsilon}=U$. The case of a general $\varepsilon>0$ will follow immediately. It is always true that $T_{U} Z \subseteq \operatorname{Ker}\left[I_{0}^{\prime \prime}(U)\right]$. We will show the converse, i.e., that if $v \in \operatorname{Ker}\left[I_{0}^{\prime \prime}(U)\right]$, namely $v$ is a solution of

$$
\begin{cases}-\Delta u-\mu \frac{u}{|x|^{2}}=\left(2^{*}(s)-1\right) \frac{U^{2^{*}(s)-2}(x)}{|x|^{s}} u, & x \in \mathbb{R}^{N} \\ u \in D_{r}^{1,2}\left(\mathbb{R}^{N}\right), \quad u>0, & x \in \mathbb{R}^{N}\end{cases}
$$

then $v \in T_{U} Z$, namely $\exists a \in \mathbb{R}$ such that $v=\left.a D_{\varepsilon} z_{\varepsilon}\right|_{\varepsilon=1}$, where $D_{\varepsilon}$ denotes the derivatives with respect to the parameter $\varepsilon$. We look for solutions $u \in D_{r}^{1,2}\left(\mathbb{R}^{N}\right)$ of problem (2.2). One has

$$
-\Psi^{\prime \prime}-\frac{n-1}{r} \Psi^{\prime}-\mu \frac{\Psi}{r^{2}}=\left(2^{*}(s)-1\right) \frac{U^{2^{*}(s)-2}}{|r|^{s}} \Psi,
$$

and then a first solution is given by

$$
w=\left.D_{\varepsilon} z_{\varepsilon}\right|_{\varepsilon=1}=C\left(r^{\frac{(2-s) \sqrt{\bar{\mu}-\mu}}{\sqrt{\bar{\mu}}}}-1\right) /\left(r^{\sqrt{\bar{\mu}}-\sqrt{\bar{\mu}-\mu}}\left(r^{\frac{(2-s) \sqrt{\bar{\mu}-\mu}}{\sqrt{\mu}}}+1\right)^{\frac{N-s}{2-s}}\right)
$$

which belongs to $D_{r}^{1,2}\left(\mathbb{R}^{N}\right)$, where $C=\sqrt{\bar{\mu}-\mu}\left(\frac{4(N-s)(\bar{\mu}-\mu)}{N-2}\right)^{\frac{N-2}{2(2-s)}}$. If we look for a second independent solution of the form $u(r)=c(r) w(r)$, we will check that $u$ is not in $D_{r}^{1,2}\left(\mathbb{R}^{N}\right)$. A direct computation gives

$$
-\left(c^{\prime \prime} w+2 c^{\prime} w^{\prime}+c w^{\prime \prime}\right)-\frac{N-1}{r}\left(c^{\prime} w+c w^{\prime}\right)-\mu \frac{c w}{r^{2}}=\left(2^{*}(s)-1\right) \frac{U^{2^{*}(s)-2}(r)}{|r|^{s}} c w,
$$

and because $w$ is a solution, we have

$$
-c^{\prime \prime} w-c^{\prime}\left(2 w^{\prime}+\frac{N-1}{r} w\right)=0 .
$$

Setting $v=c^{\prime}$, we obtain

$$
-\frac{v^{\prime}}{v}=2 \frac{w^{\prime}}{w}+\frac{N-1}{r}
$$

namely

$$
v(r)=\frac{1}{r^{N-1} w^{2}(r)} \approx C r^{1-N+2 \sqrt{\mu}-2 \sqrt{\mu}-\mu}\left(r \rightarrow 0^{+}\right),
$$

where $C$ is a constant. This implies $\quad C(r) \approx C r^{2-N+2 \sqrt{\bar{\mu}}-2 \sqrt{\mu-\mu}}$ as well as

$$
u(r) \approx C r^{\frac{2-N}{2}-\sqrt{\bar{\mu}-\mu}},
$$

as $r \rightarrow 0^{+}$. Since $\frac{2-N}{2}-\sqrt{\bar{\mu}-\mu}<0$, we have $u \notin D_{r}^{1,2}\left(\mathbb{R}^{N}\right)$. This implies a 
contradiction to assumption which had been made. So $T_{U} Z=\operatorname{Ker}\left[I_{0}^{\prime \prime}(U)\right]$. This completes the proof of Lemma.

Lemma 2.2. For all $\varepsilon>0, I_{0}^{\prime \prime}\left(z_{\varepsilon}\right)$ is a Fredholm operator with index zero. Proof. Indeed, $D_{r}^{1,2}\left(\mathbb{R}^{N}\right)$ is a Hilbert space, this implies $D_{r}^{1,2}\left(\mathbb{R}^{N}\right) \cong\left[D_{r}^{1,2}\left(\mathbb{R}^{N}\right)\right]^{*}$ and $T_{U} Z=\operatorname{Ker}\left[I_{0}^{\prime \prime}(U)\right]$, we have

$$
\begin{gathered}
I_{0}^{\prime \prime}(U): D_{r}^{1,2}\left(\mathbb{R}^{N}\right) \rightarrow\left[D_{r}^{1,2}\left(\mathbb{R}^{N}\right)\right]^{*}=D_{r}^{1,2}\left(\mathbb{R}^{N}\right) ; \\
I_{0}^{\prime \prime}(U)(v+w)=I_{0}^{\prime \prime}(U)(w), \quad \text { where } v \in T_{U} Z, w \in\left[T_{U} Z\right]^{\perp} ; \\
I_{0}^{\prime \prime}(U)(w)=-\Delta w-\mu \frac{w}{|x|^{2}}-\left(2^{*}(s)-1\right) \frac{U^{2^{*}(s)-2}(x)}{|x|^{s}} w .
\end{gathered}
$$

It is obviously that $I_{0}^{\prime \prime}(U)$ is a self-adjoint operator on $D_{r}^{1,2}\left(\mathbb{R}^{N}\right)$, we have $\left(\operatorname{Im}\left(I_{0}^{\prime \prime}(U)\right)\right)^{\perp}=T_{U} Z$, hence

$$
\operatorname{codim}\left(\operatorname{Im}\left(I_{0}^{\prime \prime}(U)\right)\right)=\operatorname{dim}\left(D_{r}^{1,2}\left(R^{N}\right) /\left[T_{U} Z\right]^{\perp}\right)=\operatorname{dim} T_{U} Z=1
$$

Moreover, fox fixed $u \in D_{r}^{1,2}\left(\mathbb{R}^{N}\right)$, the map

$$
v \mapsto \int_{\mathbb{R}^{N}} a(x) u v \mathrm{~d} x
$$

is a bounded linear functional in $D_{r}^{1,2}\left(\mathbb{R}^{N}\right)$, where $a(x)=\left(2^{*}(s)-1\right) \frac{U^{2^{*}(s)-2}(x)}{|x|^{s}}$. So by the Riesz representation theorem, there is an element in $D_{r}^{1,2}\left(\mathbb{R}^{N}\right)$, denote it by $T u$, such that

$$
\langle T u, v\rangle=\int_{\mathbb{R}^{N}} a(x) u v \mathrm{~d} x .
$$

Clearly $\quad T: D_{r}^{1,2}\left(\mathbb{R}^{N}\right) \rightarrow D_{r}^{1,2}\left(\mathbb{R}^{N}\right)$ is linear symmetric and bounded. Moreover $T$ is compact; indeed, let $\left\{u_{n}\right\}$ be a bounded sequence in $D_{r}^{1,2}\left(\mathbb{R}^{N}\right)$. Passing to a subsequence we may assume that $u_{n} \rightarrow u$ in $D_{r}^{1,2}\left(\mathbb{R}^{N}\right), u_{n} \rightarrow u$ in $L^{2^{*}(s)}\left(\mathbb{R}^{N}\right)$. Use $u$ replaced by $u_{n}-u$ and $v$ by $T u_{n}-T u$ in (2.3), and apply Hölder's inequality with $\frac{1}{2^{*}(s)}+\frac{1}{2^{*}(s)}+\frac{1}{p}=1\left(p=\frac{N-s}{2-s}\right)$ to get

$$
\begin{gathered}
\left\|T u_{n}-T u\right\|^{2} \leq\|a\|_{L^{p}}\left\|u_{n}-u\right\|_{L^{2^{*}(s)}}\left\|T u_{n}-T u\right\|_{L^{2^{*}(s)}} \\
\Rightarrow\left\|T u_{n}-T u\right\| \leq c\left\|u_{n}-u\right\|_{L^{2^{*}(s)}},
\end{gathered}
$$

which implies that $T u_{n} \rightarrow T u$ in $D_{r}^{1,2}\left(\mathbb{R}^{N}\right)$. This shows that $T$ is compact. We have

$$
\left\langle I_{0}^{\prime \prime}(U) u, v\right\rangle=\langle u, v\rangle-\langle T u, v\rangle=\langle u-T u, v\rangle=\langle(I-T) u, v\rangle .
$$

So $I_{0}^{\prime \prime}(U)=I-T$, where $I$ is an identical operator. By the fact that $\lambda I-T$ is a Fredholm operator with index zero, where $\lambda \neq 0$ and $T$ is compact, we obtain that $I_{0}^{\prime \prime}(U)=I-T$ is a Fredholm operator with index zero. This completes the 
proof of Lemma.

Now, we give the abstract perturbation method, which is crucial in our proof of the main results of this paper.

Lemma 2.3. [13] (Abstract Perturbation Method) Let $E$ be a Hilbert space and let $f_{0}, G \in C^{2}(E, \mathbb{R})$ be given. Consider the perturbed functional $f_{\varepsilon}(u)=f_{0}(u)-\varepsilon G(u)$.

Suppose that $f_{0}$ satisfies:

1) $f_{0}$ has a finite dimensional manifold of critical points $Z$, let $b=f_{0}(z)$, for all $z \in Z$;

2) for all $z \in Z, D^{2} f_{0}(z)$ is a Fredholm operator with index zero;

3) for all $z \in Z, T_{z} Z=\operatorname{Ker} D^{2} f_{0}(z)$.

Hereafter we denote by $\Gamma$ the functional $\left.G\right|_{Z}$.

Let $f_{0}$ satisfy (1)-(3) above and suppose that there exists a critical point $\bar{Z} \in Z$ of $\Gamma$ such that one of the following conditions hold:

1) $\bar{Z}$ is nondegenerated;

2) $\bar{Z}$ is a proper local minimum or maximum;

3) $\bar{Z}$ is isolated and the local topological degree of $\Gamma^{\prime}$ at $\bar{Z}, \operatorname{deg}_{\text {loc }}\left(\Gamma^{\prime}, 0\right)$ is different from zero. Then for $|\varepsilon|$ small enough, the functional $f_{\varepsilon}$ has a critical point $u_{\varepsilon}$ such that $u_{\varepsilon} \rightarrow \bar{Z}$, as $\varepsilon \rightarrow 0$.

Remark 2.4. [13] If $Z_{0}:=\left\{z \in Z: \Gamma(z)=\min _{Z} \Gamma\right\}$ is compact, then one can still prove that $f_{\varepsilon}$ has a critical point near $Z_{0}$. The set $Z_{0}$ could also consist of local minima and the same for maxima.

\section{Proof of the Theorems}

We will now solve the bifurcation equation. In order to do this, let us define the reduced functional, see [14],

$$
\begin{gathered}
\Phi_{\delta}: Z \rightarrow \mathbb{R} \\
\Phi_{\delta}\left(z_{\varepsilon}\right)=I_{\delta}\left(z_{\varepsilon}+\omega_{\delta}\left(z_{\varepsilon}\right)\right) \\
=c_{0}-\frac{\delta}{2^{*}(s)} \int_{\mathbb{R}^{N}} h(x) \frac{z_{\varepsilon}^{2^{*}(s)}(x)}{|x|^{s}} \mathrm{~d} x+o(\delta), c_{0}=I_{0}(U),
\end{gathered}
$$

where $\omega_{\delta}\left(z_{\varepsilon}\right) \perp T_{z_{\varepsilon}} Z$ and verifies $\left\|\omega_{\delta}\left(z_{\varepsilon}\right) \delta^{-1}\right\| \leq C$ as $\delta \rightarrow 0$. Hence we are led to study the finite-dimensional functional

$$
\Gamma(\varepsilon):=\int_{\mathbb{R}^{N}} h(x) \frac{z_{\varepsilon}^{2^{*}(s)}(x)}{|x|^{s}} \mathrm{~d} x=\int_{\mathbb{R}^{N}} h(\varepsilon x) \frac{U^{2^{*}(s)}(x)}{|x|^{s}} \mathrm{~d} x, \quad(\varepsilon>0) .
$$

The functional $\Gamma(\varepsilon)$ can be extended by continuity to $\varepsilon=0$ by setting

$$
\Gamma(0)=h(0) \int_{\mathbb{R}^{N}} \frac{U^{2^{*}(s)}(x)}{|x|^{s}} \mathrm{~d} x .
$$

Here we will prove the existence result by showing that problem (1.1) has a positive radial solution provided that $h$ satisfies some integrability conditions. Before giving the proof of the main results, we need the following lemma. 
Lemma 3.1. If $(\mathrm{H})$ holds, then $\Gamma_{r}(\varepsilon) \rightarrow 0$ as $\varepsilon \rightarrow+\infty$.

Proof. From the definition of $\Gamma(\varepsilon)$ and $U$, we have

$$
\begin{aligned}
\Gamma_{r}(\varepsilon) & =\int_{0}^{+\infty} h(\varepsilon r) U^{2^{*}(s)}(r) r^{N-1-s} \mathrm{~d} r \\
& =\int_{0}^{+\infty} h(\varepsilon r) \frac{((N-s)(N-2))^{\frac{N-s}{2-s}}}{\left(1+|r|^{2-s}\right)^{\frac{2(N-s)}{2-s}}} r^{N-1-s} \mathrm{~d} r \\
& =\int_{0}^{+\infty} h(r) \frac{((N-s)(N-2))^{\frac{N-s}{2-s}}}{\left(1+\left|\frac{r}{\varepsilon}\right|^{2-s}\right)^{\frac{2(N-s)}{2-s}} \frac{r^{N-1-s}}{\varepsilon^{N-s}} \mathrm{~d} r} \\
& =((N-s)(N-2))^{\frac{N-s}{2-s}} \int_{0}^{+\infty} h(r) \frac{\varepsilon^{N-s} \cdot r^{N-1-s}}{\left(\varepsilon^{2-s}+r^{2-s}\right)^{\frac{2(N-s)}{2-s}} \mathrm{~d} r} \\
& \leq C \varepsilon^{-(N-s)} \int_{0}^{1} h(r) \cdot r^{N-1-s} \mathrm{~d} r+C_{1} \varepsilon^{\alpha-(N-s)} \int_{1}^{+\infty} \frac{h(r)}{r^{\alpha}} \cdot r^{N-1-s} \mathrm{~d} r
\end{aligned}
$$

where $\alpha<N-s$. It is easy to get the first integral in the right hand side; next we show the second integral: In fact,

$$
\left(1+\left(\frac{r}{\varepsilon}\right)^{2-s}\right)^{\frac{2(N-s)}{2-s}} \cdot \frac{\varepsilon^{\alpha}}{r^{\alpha}} \geq 1 \quad(\alpha<N-s)
$$

so we have

$$
\int_{1}^{+\infty} h(r) \frac{\varepsilon^{N-s} \cdot r^{N-1-s}}{\left(\varepsilon^{2-s}+r^{2-s}\right)^{\frac{2(N-s)}{2-s}}} \mathrm{~d} r \leq \varepsilon^{\alpha-(N-s)} \int_{1}^{+\infty} \frac{h(r)}{r^{\alpha}} \cdot r^{N-1-s} \mathrm{~d} r(\alpha<N-s) .
$$

we deduce that $\Gamma_{r}(\varepsilon) \rightarrow 0$ as $\varepsilon \rightarrow+\infty$.

Proof of Theorem 1. Firstly, we claim that $\Gamma_{r}(\varepsilon)$ is not identically equal to 0 . To prove this claim we will use Fourier analysis. We introduce some notation that will be used in the following discussion. If $g \in L^{1}\left([0, \infty), \frac{\mathrm{d} r}{r}\right)$, we define

$$
M[g](s)=\int_{0}^{\infty} r^{-i s} g(r) \frac{\mathrm{d} r}{r},
$$

$M$ is nothing but the Mellin transform. The associated convolution is defined by

$$
(g \times k)(s)=\int_{0}^{\infty} g(r) k\left(\frac{s}{r}\right) \frac{\mathrm{d} r}{r} .
$$

From the definition, it follows that $M[g \times k]=M[g] \cdot M[k]$. Indeed,

$$
\begin{aligned}
& M[g(x) \times k(x)](s)=F\left[g\left(e^{x}\right) \times k\left(e^{x}\right)\right](s) \\
& =\int_{-\infty}^{+\infty}\left[g\left(e^{x}\right) \times k\left(e^{x}\right)\right] e^{-i x s} \mathrm{~d} x=\int_{-\infty}^{+\infty}\left[\int_{0}^{+\infty} g(z) k\left(\frac{e^{x}}{z}\right) \frac{\mathrm{d} z}{z}\right] e^{-i x s} \mathrm{~d} x\left(z=e^{t}\right) \\
& =\int_{-\infty}^{+\infty}\left[\int_{-\infty}^{+\infty} g\left(e^{t}\right) k\left(e^{x-t}\right) \mathrm{d} t\right] e^{-i x s} \mathrm{~d} x
\end{aligned}
$$




$$
\begin{aligned}
& =\int_{-\infty}^{+\infty} \int_{-\infty}^{\infty} g\left(e^{t}\right) e^{-i t s} k\left(e^{x-t}\right) e^{-i(x-t) s} \mathrm{~d} t \mathrm{~d} x \\
& =\int_{-\infty}^{+\infty} g\left(e^{t}\right) e^{-i t s} \mathrm{~d} t \int_{-\infty}^{\infty} k\left(e^{x-t}\right) e^{-i(x-t) s} \mathrm{~d} x \\
& =M[g(x)](s) \cdot M[k(x)](s) .
\end{aligned}
$$

With this notation we can write our $\Gamma_{r}$ in the form

$$
\Gamma_{r}(\varepsilon)=\int_{0}^{\infty} h(r) U^{2^{*}(s)}\left(\frac{r}{\varepsilon}\right)\left(\frac{r}{\varepsilon}\right)^{N-s} \frac{\mathrm{d} r}{r} .
$$

We set $m=N-s-\alpha$ and

$$
g(r)=h(r) r^{m}, k(r)=U^{2^{*}(s)}\left(\frac{1}{r}\right)\left(\frac{1}{r}\right)^{N-s-m} .
$$

Note that $g, k \in L^{1}\left([0, \infty), \frac{\mathrm{d} r}{r}\right)$. We have $\Gamma_{r}(\varepsilon)=\varepsilon^{-m}(g \times k)(\varepsilon)$ and hence if, by contradiction, $\Gamma \equiv 0$ then $g \times k \equiv 0$ and one has

$$
M[g \times k]=M[g] \cdot M[k] \equiv 0 .
$$

On the other hand, $M[k]$ is real analytic and so has a discrete number of zeros. By continuity it follows that $M[g] \equiv 0$. Then $g$ and hence $h$ are identically equal to 0 . We arrive at a contradiction. This proves the claim. Since $\Gamma_{r}(0)=0$, $\Gamma_{r}(\varepsilon) \rightarrow 0$ as $\varepsilon \rightarrow+\infty$, and $\Gamma_{r} \equiv 0$, it follows that $\Gamma_{r}$ has a maximum or a minimum at some $\bar{\varepsilon}>0$. By a straight application of Lemma 2.3 jointly with Remark 2.4, the result follows.

Proof of Theorem 2. Using Lemma 3.1, we have $\Gamma_{r}(\varepsilon) \rightarrow 0$ as $\varepsilon \rightarrow+\infty$. and $\Gamma_{r}$ can be extended to $\varepsilon=0$ by continuity setting $\Gamma_{r}(0)=a_{0} h(0)$, where $a_{0}=\int_{0}^{+\infty} U^{2^{*}(s)}(r) r^{N-1-s} \mathrm{~d} r>0$. From the assumption, we have

$$
\Gamma_{r}^{\prime}(0)=0, \Gamma_{r}^{\prime \prime}(0)=a_{1} h^{\prime \prime}(0), a_{1}=\int_{0}^{+\infty} U^{2^{*}(s)}(r) r^{N+1-s} \mathrm{~d} r>0
$$

and the condition $h(0) h^{\prime \prime}(0)>0$ implies that $\Gamma_{r}$ has a (global) maximum (if $h(0)>0$ ) or a (global) minimum (if $h(0)<0$ ), at some $\bar{\varepsilon}>0$. This allows us to use the abstract results, yielding a radial solution of problem (1.1), for $|\delta|$ small.

Proof of Theorem 3. It suffices to remark that

$$
\Gamma_{r}(1)=[(N-s)(N-2)]^{\frac{N-s}{2-s}} \int_{0}^{\infty} h(r)\left(1+r^{2-s}\right)^{-\frac{2(N-s)}{2-s}} r^{N-s-1} \mathrm{~d} r \neq 0 .
$$

If

$$
\begin{gathered}
\int_{0}^{\infty} h(r)\left(1+r^{2-s}\right)^{-\frac{2(N-s)}{2-s}} r^{N-s-1} \mathrm{~d} r>0 \\
\text { resp. } \left.\int_{0}^{\infty} h(r)\left(1+r^{2-s}\right)^{-\frac{2(N-s)}{2-s}} r^{N-s-1} \mathrm{~d} r<0\right)
\end{gathered}
$$

then $h(0) \leq 0$ (resp. $h(0) \geq 0$ ) and, once more $\Gamma_{r}$ has a (global) maximum (resp. a (global) minimum) at some $\bar{\varepsilon}>0$

In the rest of the section we will give the proof of Theorem 4 and Theorem 5 . 
First we give the following Lemma. Hypothesis ( $\left.H^{\prime}\right)$ allows us to use the following Riemann-Lebesgue convergence result.

Lemma $3.2[13]$ Let $Q=[0, T]^{N}$ be a cube in $\mathbb{R}^{N}$, and $f \in L^{q}(Q)$ be a $T$-periodic function. Consider $f_{\varepsilon}(x)=f(\varepsilon x)$, then

$$
f_{\varepsilon} \rightarrow \bar{f}=\frac{1}{|Q|} \int_{Q} f \mathrm{~d} x \text {, weakly in } L_{\text {loc }}^{q}\left(\mathbb{R}^{N}\right) \text {, as } \varepsilon \rightarrow \infty \text {. }
$$

Lemma 3.3 If ( $\left.H^{\prime}\right)$ holds, then

$$
\Gamma_{r}(\varepsilon) \rightarrow 0, \varepsilon \rightarrow+\infty .
$$

Proof. Given $\varepsilon>0$, there exists $R>0$ large enough such that

$$
\begin{aligned}
& \left|\int_{R}^{\infty} h(r) z_{\varepsilon}^{2^{*}(s)}(r) r^{N-s-1} \mathrm{~d} r\right| \\
& \leq\|h(r)\|_{\infty} \int_{R}^{\infty} z_{\varepsilon}^{2^{*}(s)}(r) r^{N-s-1} \mathrm{~d} r<\varepsilon .
\end{aligned}
$$

On the other hand, the remainder integral over the interval $0 \leq r<R$ tends to 0 as $\varepsilon \rightarrow \infty$ because of hypothesis $\left(\mathrm{H}^{\prime}\right)$ and the Riemann-Lebesgue lemma.

Proof of Theorem 4. Using Lemma 3.3, we have $\Gamma_{r}(\varepsilon) \rightarrow 0$ as $\varepsilon \rightarrow+\infty$. and $\Gamma_{r}$ can be extended to $\varepsilon=0$ by continuity setting $\Gamma_{r}(0)=a_{0} h(0)$, where $a_{0}=\int_{0}^{+\infty} U^{2^{*}(s)}(r) r^{N-1-s} \mathrm{~d} r>0$. From the assumption, we have

$$
\Gamma_{r}^{\prime}(0)=0, \Gamma_{r}^{\prime \prime}(0)=a_{1} h^{\prime \prime}(0), a_{1}=\int_{0}^{+\infty} U^{2^{*}(s)}(r) r^{N+1-s} \mathrm{~d} r>0 .
$$

and the condition $h(0) h^{\prime \prime}(0)>0$ implies that $\Gamma_{r}$ has a (global) maximum (if $h(0)>0$ ) or a (global) minimum (if $h(0)<0$ ), at some $\bar{\varepsilon}>0$. This allows us to use the abstract results, yielding a radial solution of problem (1.1), for $|\delta|$ small.

Proof of Theorem 5. It suffices to repeat the arguments used to prove Theorem 1 using Lemma 3.1 instead of Lemma 3.3.

\section{Conclusion}

We study a class of semilinear elliptic problems involving critical Hardy-Sobolev exponent and Hardy terms, and obtain positive radial solutions for these problems via an abstract perturbation method in critical point theory. Extensions of nonradial solutions for these problems are being investigated by the author. Results will be submitted for publication in the near future.

\section{Acknowledgements}

We would like to thank the editor and the referee for their valuable comments which have led to an improvement of the presentation of this paper.

\section{Fund}

This work is supported by Natural Science Foundation of China (No. 11671331); Natural Science Foundation of Fujian Province (No. 2015J01585) and Scientific Research Foundation of Jimei University. 


\section{References}

[1] Garcia Azorero, J.P. and Peral Alonso, I. (1998) Hardy Inequalities and Some Critical Elliptic and Parabolic Problems. Journal of Differential Equations, 144, 441-476. https://doi.org/10.1006/jdeq.1997.3375

[2] Palais, R.S. (1979) The Principle of Symmetric Criticality. Communications in Mathematical Physics, 69, 19-30. https://doi.org/10.1007/BF01941322

[3] Li, Y.Y., Ruf, B., Guo, Q.Q. and Niu, P.C. (2014) Positive Solutions for Singular Elliptic Equations with Mixed Dirichlet-Neumann Boundary Conditions. Mathematische Nachrichten, 287, 374-397. https://doi.org/10.1002/mana.201100351

[4] Bouchekif, M. and Messirdi, S. (2015) On Elliptic Problems with Two Critical Hardy-Sobolev Exponents at the Same Pole. Applied Mathematics Letters, 42, 9-14. https://doi.org/10.1016/j.aml.2014.10.012

[5] Lan, Y.Y. and Tang, C.L. (2014) Perturbation Methods in Semilinear Elliptic Problems Involving Critical Hardy-Sobolev Exponent. Acta Mathematica Scientia, 34B, 703-712. https://doi.org/10.1016/S0252-9602(14)60041-2

[6] Yan, S.S. and Yang, J.F. (2013) Infinitely Many Solutions for an Elliptic Problem Involving Critical Sobolev and Hardy-Sobolev Exponents. Calculus of Variations and Partial Differential Equations, 48, 587-610.

https://doi.org/10.1007/s00526-012-0563-7

[7] Ding, L. and Tang, C.L. (2008) Existence and Multiplicity of Positive Solutions for a Class of Semilinear Elliptic Equations Involving Hardy Term and Hardy-Sobolev Critical Exponents. Journal of Mathematical Analysis and Applications, 339, 1073-1083. https://doi.org/10.1016/j.jmaa.2007.07.066

[8] Shang, Y.Y. and Tang, C.L. (2009) Positive Solutions for Neumann Elliptic Problems Involving Critica Hardy-Sobolev Exponent with Boundary Singularities. Nonlinear Analysis: Theory, Methods \& Applications, 70, 1302-1320.

https://doi.org/10.1016/j.na.2008.02.013

[9] Cao, D.M., He, X.M. and Peng, S.J. (2005) Positive Solutions for Some Singular Critical Growth Nonlinear Elliptic Equations. Nonlinear Analysis. Theory, Methods \& Applications, 60, 589-609. https://doi.org/10.1016/j.na.2004.08.042

[10] Cao, D.M. and Peng, S.J. (2003) A Note on the Sign-Changing Solutions to Elliptic Problems with Critical Sobolev and Hardy Terms. Journal of Differential Equations, 193, 424-434. https://doi.org/10.1016/S0022-0396(03)00118-9

[11] Ghoussoub, N. and Kang, X.S. (2004) Hardy-Sobolev Critical Elliptic Equations with Boundary Singularities. Annales de P Institut Henri Poincaré C, Analyse non linéaire, 21, 767-793.

[12] Kang, D.S. and Peng, S.J. (2005) Solutions for Semi-Linear Elliptic Problems with Critical Hardy-Sobolev Exponents and Hardy Potential. Applied Mathematics Letters, 18, 1094-1100.

[13] Wang, C. and Shang, Y.Y. (2017) Existence and Multiplicity of Positive Solutions for Elliptic Equation with Critical Weighted Hardy-Sobolev Exponents and Boundary Singularities. Computers Mathematics with Applications, 74, 701-713.

[14] Wang, C. and Shang, Y.Y. (2017) Existence and Multiplicity of Positive Solutions for a Perturbed Semilinear Elliptic Equation with Two Hardy-Sobolev Critical Exponents. Journal of Mathematical Analysis and Applications, 451, 1198-1215.

[15] Bhakta, M. (2017) Infinitely Many Sign-Changing Solutions of an Elliptic Problem Involving Critical Sobolev and Hardy-Sobolev Exponent. Proceedings of the Indian Academy of Sciences-Mathematical Sciences, 127, 337-347. 
https://doi.org/10.1007/s12044-016-0304-5

[16] Deng, Z.Y. and Huang, Y.S. (2017) Positive Symmetric Results for a Weighted Quasilinear Elliptic System with Multiple Critical Exponents in $\mathrm{R}^{\mathrm{N}}$, Boundary Value Problems. https://doi.org/10.1186/s13661-017-0758-0

[17] Ambrosetti, A., Garcia Azorero, J. and Peral, I. (1999) Perturbation of $\Delta u+u^{\frac{N+2}{N-2}}=0$, the Scalar Curvature Problem in $\mathrm{R}^{\mathrm{N}}$, and Related Topics. Journal of Functional Analysis, 165, 117-149. https://doi.org/10.1006/jfan.1999.3390

[18] Ambrosetti, A. and Malchiodi, A. (2006) Perturbation Methods and Semilinear Elliptic Problems on $\mathrm{R}^{\mathrm{n}}$. Birkhäuser Verlag. 